\title{
Quasistationary problem for a cracked body with electrothermoconductivity
}

\author{
D. HÖMBERG ${ }^{\dagger}$ \\ Weierstrass Institute for Applied Analysis und Stochastics, Mohrenstraße 39, D-10117 Berlin, \\ Germany \\ A. M. KHLUDNEV \\ Lavrentyev Institute of Hydrodynamics of the Russian Academy of Sciences, Novosibirsk \\ 630090, Russia \\ AND \\ J. SOKOŁOWSKI \\ Institut Elie Cartan, Laboratoire de Mathématiques, Université Henri Poincaré Nancy I, \\ BP 239, 54506 Vandoeuvre lès Nancy Cedex, France
}

[Received 10 September 1999 and in revised form 25 October 2000]

\begin{abstract}
We consider a problem related to resistance spot welding. The mathematical model describes the equilibrium state of an elastic, cracked body subjected to heat transfer and electroconductivity and can be viewed as an extension to the classical thermistor problem.

We prove existence of a solution in Sobolev spaces.
\end{abstract}

Keywords: Crack; thermistor; thermoelastic contact; spot welding

\section{Introduction}

In resistance spot welding two workpieces are pressed together by electrodes. Owing to the Joule effect and the high resistivity in the contact area between the workpieces, the welding current leads to an increase in temperature, until finally a weld nugget is formed (see Fig. 1).

For a complete description of the process, one has to take into account mechanical, thermal and electrical effects, as well as the free boundary between liquid metal and solid. To the knowledge of the authors, mathematical models up to now have only considered the thermal and electrical effects, neglecting mechanics (see, for example, [15]).

A mathematical model for the special case of impulse resistance welding has been developed in [7]. The basic equations to obtain the displacement $u=\left(u_{1}, u_{2}\right)$, the temperature $\theta$ and the electric potential $\varphi$ are the quasistatic balance law of momentum, the balance of internal energy as well as the quasistatic balance law of electrical charge. In the framework of isotropic linearized thermoelasticity, we can formulate the balance laws in the undeformed domain.

\footnotetext{
†Email: hoemberg@wias-berlin.de

*Email: khlud@hydro.nsc.ru

${ }^{\S}$ Email: sokolows@iecn.u-nancy.fr
} 


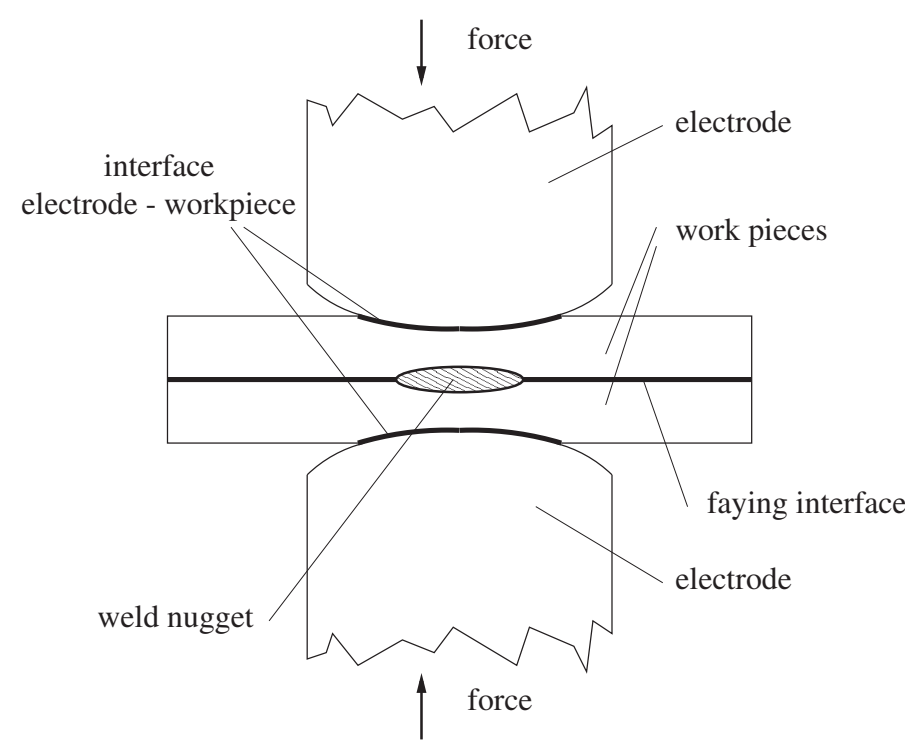

FIG. 1. Schematic illustration of the resistance spot welding process.

Using Ohm's law

$$
J=\gamma(\theta) E,
$$

where $J$ is the current density, $E$ the electric field and $\gamma(\theta)$ the electric conductivity, and

$$
E=-\nabla \varphi
$$

the quasistatic balance law of electrical charge reads

$$
-\operatorname{div}(\gamma(\theta) \nabla \varphi)=0 .
$$

We tacitly assume that the density, the specific heat and the heat conductivity are independent of temperature and normalized to one. Then the first two balance laws lead to the following system of partial differential equations:

$$
\begin{aligned}
-\sigma_{i j, j}+\delta^{2} \theta_{, i} & =0, \quad i=1,2, \\
\theta_{t}-\Delta \theta+\delta^{2} \bar{\theta} \frac{\partial}{\partial t} \operatorname{div} u & =J_{i} E_{i},
\end{aligned}
$$

where $\bar{\theta}$ is the temperature in the stress-free state and $\delta$ describes the thermal expansion ( [6], pp. 48$51,[13])$. The elastic part of the stress tensor, $\sigma_{i j}, i, j=1,2$, is given by Hooke's law

$$
\sigma_{i j}=a_{i j k l} \varepsilon_{k l}(u)
$$

where $\varepsilon_{k l}(u)=\frac{1}{2}\left(u_{k, l}+u_{l, k}\right)$ are the strain tensor components. In what follows we put $\bar{\theta}=1$ without any loss of generality. The source term $J_{i} E_{i}$ corresponds to the Joule heat caused by the electric current. Using Ohm's law, it can be rewritten as

$$
J_{i} E_{i}=\gamma(\theta)|\nabla \varphi|^{2} .
$$




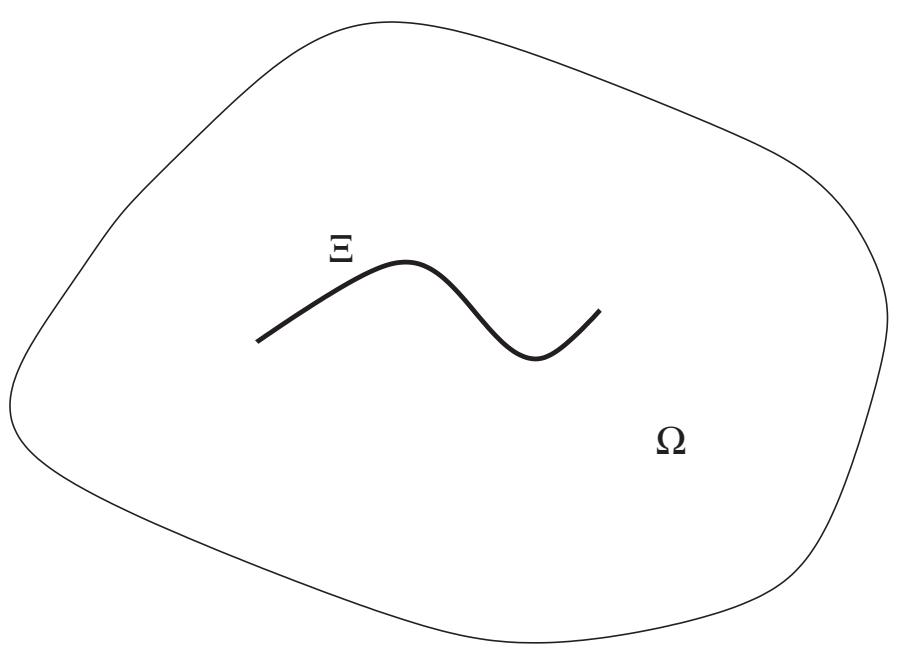

FIG. 2. The domain $\Omega_{c}$.

Obviously, the most important control parameters for the process are the force applied to join the workpieces and the shape of the electrode (Fig. 1). To achieve a uniform current density between the electrodes, flat electrodes would be desirable. On the other hand, to reduce wear, a domed electrode is more favourable. Hence, the area of contact between electrode and workpiece is very important to control the quality of the weld joint.

The aim of this paper is to initiate the investigation of this contact problem. Owing to the quadratic Joule heating term in the energy balance, a crucial point for the analysis will be the regularity of solutions for the electric potential equation. To avoid the additional difficulties which arise from the geometric singularity at the boundary of the contact between electrode and workpiece, we focus on the simplified problem of a cracked thermoelectroelastic body.

Note that the so-called thermistor problem for finding the temperature and electrical potential was considered in $[4,5,8,19,20]$. The Stefan problem with Joule's heating was analysed in [15]. On the other hand, there are many papers related to equilibrium of elastic bodies with cracks and nonpenetration conditions imposed on the crack faces (see $[9,11,12]$ ), and to thermoelastic bodies with linear and nonlinear boundary conditions of Signorini's type (see $[1,3,14,18]$ ).

In the next section we give a precise formulation of the model, assumptions and the main result are stated in Section 3 and the proof is given in Section 4.

\section{Mathematical model and main result}

Let $\Omega \subset \mathbb{R}^{2}$ be a bounded domain with smooth boundary $\Gamma$, and $\Xi \subset \Omega$ be a smooth curve without self-intersections. Denote $\Omega_{c}=\Omega \backslash \bar{\Xi}, Q_{c}=\Omega_{c} \times(0, T), Q=\Omega \times(0, T), T>0$. Assume that $\Gamma=\Gamma_{1} \cup \Gamma_{2}, \Gamma_{1} \cap \Gamma_{2}=\emptyset$, meas $\Gamma_{1}>0$.

In the domain $Q_{c}$ (see Fig. 2), we want to find a solution $u=\left(u_{1}, u_{2}\right), \theta, \varphi$ of the following 
boundary value problem:

$$
\begin{aligned}
& -\sigma_{i j, j}+\delta^{2} \theta_{i}=0, \quad i=1,2, \\
& \theta_{t}-\Delta \theta+\delta^{2} \frac{\partial}{\partial t} \operatorname{div} u=\gamma(\theta)|\nabla \varphi|^{2}, \\
& \operatorname{div}(\gamma(\theta) \nabla \varphi)=0 \\
& \theta=\theta_{0} \quad \text { for } t=0 \text {, } \\
& \varphi=\varphi_{0}, \quad \theta=0 \quad \text { on } \Gamma \times(0, T), \\
& \sigma_{i j} n_{j}=g_{i} \text { on } \Gamma_{2} \times(0, T), \quad i=1,2, \\
& {[\varphi]=\left[\gamma(\theta) \frac{\partial \varphi}{\partial v}\right]=0, \quad[\theta]=\left[\frac{\partial \theta}{\partial v}\right]=0 \quad \text { on } \Xi \times(0, T),} \\
& u=0 \quad \text { on } \Gamma_{1} \times(0, T) ; \quad[u] \cdot v \geqslant 0 \quad \text { on } \Xi \times(0, T), \\
& \sigma_{\nu} \leqslant 0, \quad\left[\sigma_{v}\right]=0, \quad \sigma_{\tau}=0, \quad \sigma_{v} \cdot[u] \cdot v=0 \quad \text { on } \Xi \times(0, T) .
\end{aligned}
$$

We select a unit normal vector $v=\left(v_{1}, v_{2}\right)$ to $\Xi$, and $n=\left(n_{1}, n_{2}\right)$ is a unit normal vector to $\Gamma,\left\{\sigma_{i j} v_{j}\right\}=\sigma_{\tau}+\sigma_{v} \cdot v, i=1,2, \sigma_{v}=\sigma_{i j} v_{j} v_{i}, \tau=\left(-v_{2}, v_{1}\right)$. The mathematical model (2.1)-(2.9) describes the equilibrium state of an elastic body subjected to the heat transfer and electroconductivity. The brackets $[v]=v^{+}-v^{-}$mean the jump of $v$ across $\Xi$ and $v^{+}, v^{-}$ stand for the values of $v$ on $\Xi^{+}, \Xi^{-}$, respectively, where $\Xi^{+}, \Xi^{-}$are defined for given choice of positive and negative directions of $v$ on $\Xi$. The curve $\Xi$ presents the crack in the body, and the second inequality of (2.8) corresponds to the mutual nonpenetration condition between the crack faces.

Note that the jump conditions in (2.7) are consistent with the framework of linearized elasticity, since we work in the reference domain.

We make the following assumptions: $\gamma$ is a given continuous function, $\gamma_{1} \leqslant \gamma(s) \leqslant \gamma_{2}$, $s \in R ; \gamma_{1}, \gamma_{2}$ are positive constants. The elastic coefficients $a_{i j k l}$ are smooth and satisfy the usual assumptions of symmetry and positive definiteness. For the boundary and initial data we assume $\theta_{0} \in H_{0}^{1}(\Omega) ; g_{i} \in H^{1}\left(0, T ; L^{2}\left(\Gamma_{2}\right)\right), i=1,2 ; \varphi_{0} \in L^{\infty}\left(0, T ; H^{\frac{3}{2}}(\Gamma)\right)$.

Here

$$
H_{0}^{1}(\Omega)=\left\{v \in H^{1}(\Omega) \mid v=0 \text { on } \Gamma\right\} .
$$

The space $H^{\frac{3}{2}}(\Gamma)$ can be defined as the space of traces on $\Gamma$ of all functions from $H^{2}(\Omega)$. To formulate an existence theorem we first have to note that for any given $g=\left(g_{1}, g_{2}, g_{3}\right) \in L^{p}(\Omega)$, $p>1$, the solution $w$ of the problem

$$
\begin{aligned}
\operatorname{div}(\nabla w+g) & =0 \quad \text { in } \quad \Omega, \\
w & =0 \quad \text { on } \quad \Gamma
\end{aligned}
$$

exists and the following estimate holds:

$$
\|\nabla w\|_{L^{p}(\Omega)} \leqslant \Lambda_{p}\|g\|_{L^{p}(\Omega)}
$$

with the positive constant $\Lambda_{p}$ depending on $p$. Assume that the oscillation of the function $\gamma$ is small enough so that

$$
\Lambda<1, \quad \Lambda=\frac{\gamma_{2}-\gamma_{1}}{\gamma_{1}+\gamma_{2}} \Lambda_{4}
$$


With this merely technical assumption, we can formulate the following existence theorem.

THEOREM 2.1 Assume that all assumptions concerning $g_{i}, \gamma, \varphi_{0}, \theta_{0}, a_{i j k l}$ are satisfied. Then for small $\delta$ there exists a solution to the problem (2.1)-(2.9) such that

$$
\begin{aligned}
& \theta_{t} \in L^{2}\left(Q_{c}\right), \quad \theta \in L^{2}\left(0, T ; H^{1}\left(\Omega_{c}\right)\right), \quad u \in K, u \in H^{1}\left(0, T ; H^{1}\left(\Omega_{c}\right)\right), \\
& \varphi \in L^{\infty}\left(0, T ; W_{4}^{1}(\Omega)\right), \\
& \int_{Q_{c}} \sigma_{i j} \varepsilon_{i j}(\bar{u}-u)-\delta^{2} \int_{Q_{c}} \theta \operatorname{div}(\bar{u}-u) \geqslant \int_{\Gamma_{2} \times(0, T)} g_{i}\left(\bar{u}_{i}-u_{i}\right) \quad \forall \bar{u} \in K, \\
& \int_{Q_{c}}\left(\theta_{t}+\delta^{2} \frac{\partial}{\partial t} \operatorname{div} u-\gamma(\theta)|\nabla \varphi|^{2}\right) \eta=-\int_{Q_{c}} \nabla \theta \cdot \nabla \eta \forall \eta \in L^{2}\left(0, T ; H_{0}^{1}(\Omega)\right), \\
& \int_{Q} \gamma(\theta) \nabla \varphi \cdot \nabla \psi=0 \forall \psi \in L^{2}\left(0, T ; H_{0}^{1}(\Omega)\right) .
\end{aligned}
$$

Here

$$
K=\left\{v \in L^{2}\left(0, T ; H^{1}\left(\Omega_{c}\right)\right) \mid v=0 \text { on } \Gamma_{1} \times(0, T),[v] \cdot v \geqslant 0 \text { on } \Xi \times(0, T)\right\} .
$$

\section{Proof of Theorem 2.1}

To prove the existence of a solution to (2.1)-(2.9) we substitute the function $\theta=\bar{\theta}$ in (2.3) and determine the function $\varphi$ from (2.3) and the first conditions of (2.5), (2.7), respectively. Then we consider $\gamma(\bar{\theta})|\nabla \varphi|^{2}$ as a given function in the right-hand side of (2.2) and solve the equations (2.1), (2.2) along with all boundary and initial conditions. In such a way we find the functions $u, \theta$. The next step of the proof is to show that the mapping $A: \bar{\theta} \longrightarrow \theta$ admits a fixed point in an appropriate functional space. To this end we use the Schauder fixed point theorem.

Let $\bar{\theta} \in L^{2}\left(0, T ; L^{2}\left(\Omega_{c}\right)\right)$ be any fixed function. Consider the following problem:

$$
\begin{aligned}
& \operatorname{div}(\gamma(\bar{\theta}) \nabla \varphi)=0 \quad \text { in } \quad Q_{c}, \\
& \varphi=\varphi_{0} \quad \text { on } \quad \Gamma \times(0, T), \\
& {[\varphi]=0, \quad\left[\gamma(\bar{\theta}) \frac{\partial \varphi}{\partial \nu}\right]=0 \quad \text { on } \quad \Xi \times(0, T) .}
\end{aligned}
$$

Here, $t$ plays the role of a parameter. Note first that the conditions $\xi \in H^{1}\left(\Omega_{c}\right),[\xi]=0$ on $\Xi$ provide the inclusion $\xi \in H^{1}(\Omega)$.

Consider the problem (3.1), (3.2) with the first condition of (3.3). The weak solution of this problem can be defined as

$$
\begin{array}{ll}
\varphi \in L^{\infty}\left(0, T ; H^{1}(\Omega)\right), \\
\int_{Q} \gamma(\bar{\theta}) \nabla \varphi \cdot \nabla \psi=0 \quad \forall \psi \in L^{2}\left(0, T ; H_{0}^{1}(\Omega)\right)
\end{array}
$$

with the condition (3.2). It is easy to obtain the estimate for the function $\varphi$ by choosing $\psi=\varphi-\Phi_{0}$. Here we take $\Phi_{0}$ as an element of the space $L^{\infty}\left(0, T ; H^{2}(\Omega)\right)$ such that $\Phi_{0}=\varphi_{0}$ on $\Gamma \times(0, T)$. 
From the condition imposed on $\varphi_{0}$ it follows that such an extension of $\varphi_{0}$ in the domain $Q$ exists. As a result of the substitution we have the equality

$$
\int_{Q} \gamma(\bar{\theta}) \nabla \varphi \cdot\left(\nabla \varphi-\nabla \Phi_{0}\right)=0
$$

which provides the estimate

$$
\gamma_{1} \int_{Q}|\nabla \varphi|^{2} \leqslant \gamma_{2} \int_{Q}\left|\nabla \varphi \cdot \nabla \Phi_{0}\right|
$$

Hence the above inclusion $\varphi \in L^{\infty}\left(0, T ; H^{1}(\Omega)\right)$ follows. Existence of the solution is proved by the standard variational method. Moreover, the second condition of (3.3) is fulfilled since equation (3.1) holds in $Q$ (compare [9] and [11]).

Indeed, in the domain $Q$, consider the zeroth distribution $\operatorname{div}(\gamma(\bar{\theta}) \nabla \varphi)$. Denote by $\langle\cdot, \xi\rangle$ the value of a distribution at the test function $\xi$. We divide $\Omega_{c}$ into two subdomains $\Omega_{1}, \Omega_{2}$ by extending the curve $\Xi$. In doing so we assume that the extended curve crosses the boundary $\Gamma$ at two points, and the boundaries $\partial \Omega_{i}, i=1,2$, with unit external normals $v^{1}, v^{2}$, respectively, to possess the Lipschitz property, $\Gamma_{1} \cap \partial \Omega_{i} \neq \emptyset, i=1,2$. We have in $Q$,

$$
\langle\operatorname{div}(\gamma(\bar{\theta}) \nabla \varphi), \xi\rangle=0, \quad \xi \in C_{0}^{\infty}(Q) .
$$

Consequently,

$$
\begin{aligned}
\langle\operatorname{div}(\gamma(\bar{\theta}) \nabla \varphi), \xi\rangle & =-\int_{\Omega_{1} \times(0, T)} \gamma(\bar{\theta}) \nabla \varphi \cdot \nabla \xi-\int_{\Omega_{2} \times(0, T)} \gamma(\bar{\theta}) \nabla \varphi \cdot \nabla \xi \\
& =\int_{0}^{T}\left\langle\left[\gamma(\bar{\theta}) \frac{\partial \varphi}{\partial v}\right], \xi\right\rangle_{\Xi, 1 / 2} \mathrm{~d} t=0 .
\end{aligned}
$$

Here we use the following well known fact. Let $D \subset R^{2}$ be a bounded domain with a Lipschitz boundary $\partial D$. Then the conditions $u \in H^{1}(D), \operatorname{div}(a \nabla u) \in L^{2}(D), a \in L^{\infty}(D)$ imply $a \frac{\partial u}{\partial v} \in$ $H^{-1 / 2}(\partial D)$, and the Green formula holds: i.e.

$$
\int_{D} \operatorname{div}(a \nabla u) \xi=\left\langle a \frac{\partial u}{\partial n}, \xi\right\rangle_{1 / 2}-\int_{D} a \nabla u \cdot \nabla \xi \quad \forall \xi \in H^{1}(D),
$$

where $\langle\cdot, \cdot\rangle_{1 / 2}$ is the duality pairing between $H^{-1 / 2}(\partial D)$ and $H^{1 / 2}(\partial D)$. This implies the second condition of (3.3),

$$
\int_{0}^{T}\left\langle\left[\gamma(\bar{\theta}) \frac{\partial \varphi}{\partial v}\right], \xi\right\rangle_{\Xi, 1 / 2} \mathrm{~d} t=0
$$

which holds in the sense

$$
\int_{0}^{T}\left\langle\gamma(\bar{\theta}) \frac{\partial \varphi}{\partial \nu^{1}}, \xi\right\rangle_{\partial \Omega_{1}, 1 / 2} \mathrm{~d} t+\int_{0}^{T}\left\langle\gamma(\bar{\theta}) \frac{\partial \varphi}{\partial \nu^{2}}, \xi\right\rangle_{\partial \Omega_{2}, 1 / 2} \mathrm{~d} t=0, \quad \xi \in C_{0}^{\infty}(Q) .
$$

Hence we obtain the following boundary value problem for $\varphi$ :

$$
\begin{gathered}
\operatorname{div}(\gamma(\bar{\theta}) \nabla \varphi)=0 \quad \text { in } \quad Q, \\
\varphi=\varphi_{0} \quad \text { on } \quad \Gamma \times(0, T) .
\end{gathered}
$$


Now we aim to show an additional regularity for $\varphi$. The problem (3.5), (3.6) can be rewritten in the form

$$
\begin{array}{r}
\operatorname{div}\left(\frac{\gamma_{1}+\gamma_{2}}{2} \nabla v+\tilde{\gamma}(\bar{\theta}) \nabla v+\gamma(\bar{\theta}) \nabla \Phi_{0}\right)=0 \quad \text { in } \quad Q, \\
v=0 \quad \text { on } \quad \Gamma \times(0, T) .
\end{array}
$$

Here $v=\varphi-\Phi_{0}$ is unknown function, $\tilde{\gamma}(s)=\gamma(s)-\frac{\gamma_{1}+\gamma_{2}}{2}$. Note that $|\tilde{\gamma}(s)| \leqslant \frac{\gamma_{2}-\gamma_{1}}{2}, s \in \mathbb{R}$.

Take any function $v^{0} \in L^{\infty}\left(0, T ; W_{4}^{1}(\Omega)\right)$ and apply the iteration method for solving the problem (3.7), (3.8):

$$
\begin{array}{r}
\operatorname{div}\left(\frac{\gamma_{1}+\gamma_{2}}{2} \nabla v^{n+1}+\tilde{\gamma}(\bar{\theta}) \nabla v^{n}+\gamma(\bar{\theta}) \nabla \Phi_{0}\right)=0 \quad \text { in } \quad Q, \\
v^{n+1}=0 \quad \text { on } \quad \Gamma \times(0, T),
\end{array}
$$

where $n=0,1,2, \ldots$ According to (2.10) for almost all $t \in(0, T)$ we have the estimate

$$
\left\|\nabla v^{n+1}\right\|_{L^{4}(\Omega)} \leqslant \Lambda\left\|\nabla v^{n}\right\|_{L^{4}(\Omega)}+\lambda\left\|\nabla \Phi_{0}\right\|_{L^{4}(\Omega)}, \quad \lambda=\frac{2 \gamma_{2} \Lambda_{4}}{\gamma_{1}+\gamma_{2}} .
$$

By (3.11), it is easy to conclude that the sequence $v^{n}$ is fundamental, and we can assume that as $n \rightarrow \infty$

$$
v^{n} \rightarrow v \quad \text { in } \quad L^{\infty}\left(0, T ; L^{4}(\Omega)\right) .
$$

This allows us to pass to the limit in (3.9), (3.10) as $n \rightarrow \infty$. Hence the problem (3.7), (3.8) (or, what is the same, the problem (3.5), (3.6)) has the solution $v \in L^{\infty}\left(0, T ; W_{4}^{1}(\Omega)\right.$ ), i.e. $\varphi \in L^{\infty}\left(0, T ; W_{4}^{1}(\Omega)\right)$. Consequently, $\nabla \varphi \in L^{\infty}\left(0, T ; L^{4}(\Omega)\right)$. This implies that $\gamma(\bar{\theta})|\nabla \varphi|^{2} \in$ $L^{2}\left(Q_{c}\right)$, and we can consider the following initial-boundary value problem in $Q_{c}$ for unknown functions $u=\left(u_{1}, u_{2}\right), \theta$ :

$$
\begin{array}{rrr}
-\sigma_{i j, j}+\delta^{2} \theta_{, i}=0, & i=1,2, \\
\theta_{t}-\Delta \theta+\delta^{2} \frac{\partial}{\partial t} \operatorname{div} u=\gamma(\bar{\theta})|\nabla \varphi|^{2}, & \\
{[u] \cdot v \geqslant 0, \quad \sigma_{v} \leqslant 0, \quad\left[\sigma_{v}\right]=0, \quad \sigma_{\tau}=0, \quad \sigma_{v} \cdot[u] \cdot v=0} & \text { on } \Xi \times(0, T), \\
\theta=0 & \text { on } \Gamma \times(0, T), \\
{[\theta]=\left[\frac{\partial \theta}{\partial v}\right]=0} & \text { on } \Xi \times(0, T), \\
\theta=\theta_{0} \quad & \text { for } t=0 .
\end{array}
$$

The problem (3.12)-(3.18) with the given right-hand side $h=\gamma(\bar{\theta})|\nabla \varphi|^{2} \in L^{2}\left(Q_{c}\right)$ can be solved for small $\delta$ (see $[10,14]$ ), with the following estimates:

$$
\begin{array}{r}
\left\|\theta_{t}\right\|_{L^{2}\left(Q_{c}\right)}+\|\theta\|_{L^{2}\left(0, T ; H^{1}\left(\Omega_{c}\right)\right)} \leqslant c_{1} \delta\|u\|_{H^{1}\left(0, T ; H^{1}\left(\Omega_{c}\right)\right)}+c_{2}\|h\|_{L^{2}\left(Q_{c}\right)}, \\
\|u\|_{H^{1}\left(0, T ; H^{1}\left(\Omega_{c}\right)\right)} \leqslant c_{3} \delta\|\theta\|_{H^{1}\left(Q_{c}\right)}+c_{4}\|g\|_{H^{1}\left(0, T ; L^{2}\left(\Gamma_{2}\right)\right)}+c_{5}\left\|\theta_{0}\right\|_{H^{1}(\Omega)},
\end{array}
$$


and the constants $c_{i}$ are independent of $\delta, \bar{\theta}$. This solution $(u, \theta)$ satisfies the variational inequality

$$
\int_{Q_{c}} \sigma_{i j}(u) \varepsilon_{i j}(\bar{u}-u)-\delta^{2} \int_{Q_{c}} \theta \operatorname{div}(\bar{u}-u) \geqslant \int_{\Gamma_{2} \times(0, T)} g_{i}\left(\bar{u}_{i}-u_{i}\right) \quad \forall \bar{u} \in K,
$$

and the identity

$$
\int_{Q_{c}}\left(\theta_{t}+\delta^{2} \frac{\partial}{\partial t} \operatorname{div} u-\gamma(\bar{\theta})|\nabla \varphi|^{2}\right) \eta=-\int_{Q_{c}} \nabla \theta \cdot \nabla \eta \quad \forall \eta \in L^{2}\left(0, T ; H_{0}^{1}(\Omega)\right) .
$$

Note that inequality (3.21) can be written for almost all $t \in(0, T)$ in the following form:

$$
\int_{\Omega_{c}} \sigma_{i j}(u) \varepsilon_{i j}(\bar{u}-u)-\delta^{2} \int_{\Omega_{c}} \theta \operatorname{div}(\bar{u}-u) \geqslant \int_{\Gamma_{2}} g_{i}\left(\bar{u}_{i}-u_{i}\right) \quad \forall \bar{u} \in \tilde{K},
$$

where

$$
\tilde{K}=\left\{v \in H^{1}\left(\Omega_{c}\right) \mid v=0 \text { on } \Gamma_{1}, \quad[v] \cdot v \geqslant 0 \quad \text { on } \Xi\right\} .
$$

The presence of the estimates (3.19), (3.20) allows us to find $\delta_{0}$ such that for all $\delta \leqslant \delta_{0}$ the problem (3.12)-(3.18) is solvable. The bound $\delta_{0}$ for $\delta$ can be found similar to [10]. The bound depends on the norms of $\theta_{0}, \varphi_{0}, g$ in the respective spaces and on the domain $\Omega_{c}$ (dependence on $\varphi_{0}$ appears due to the presence of $\gamma(\bar{\theta})|\nabla \varphi|^{2}$ on the right-hand side of (3.19)).

In what follows we fix any $\delta \leqslant \delta_{0}$ which provides the solvability of (3.12)-(3.18). Notice that the solution $(\theta, u)$ is unique. Indeed, assume that there are two solutions $\left(\theta^{1}, u^{1}\right),\left(\theta^{2}, u^{2}\right)$, corresponding to the function $h$. These solutions satisfy the relations

$$
\begin{gathered}
\theta_{t}^{1}-\Delta \theta^{1}+\delta^{2} \frac{\partial}{\partial t} \operatorname{div} u^{1}=h, \\
\int_{\Omega_{c}} \sigma_{i j}\left(u^{1}\right) \varepsilon_{i j}\left(\bar{u}-u^{1}\right)-\delta^{2} \int_{\Omega_{c}} \theta^{1} \operatorname{div}\left(\bar{u}-u^{1}\right) \geqslant \int_{\Gamma_{2}} g_{i}\left(\bar{u}_{i}-u_{i}^{1}\right) \quad \forall \bar{u} \in \tilde{K}, \\
\theta_{t}^{2}-\Delta \theta^{2}+\delta^{2} \frac{\partial}{\partial t} \operatorname{div} u^{2}=h, \\
\int_{\Omega_{c}} \sigma_{i j}\left(u^{2}\right) \varepsilon_{i j}\left(\bar{u}-u^{2}\right)-\delta^{2} \int_{\Omega_{c}} \theta^{2} \operatorname{div}\left(\bar{u}-u^{2}\right) \geqslant \int_{\Gamma_{2}} g_{i}\left(\bar{u}_{i}-u_{i}^{2}\right) \quad \forall \bar{u} \in \tilde{K} .
\end{gathered}
$$

Denote $\theta=\theta^{1}-\theta^{2}, u=u^{1}-u^{2}$. From (3.24)-(3.27) it follows that

$$
\begin{gathered}
\theta_{t}-\Delta \theta+\delta^{2} \frac{\partial}{\partial t} \operatorname{div} u=0 \\
\int_{\Omega_{c}} \sigma_{i j}(u) \varepsilon_{i j}(u)-\delta^{2} \int_{\Omega_{c}} \theta \operatorname{div} u \leqslant 0 .
\end{gathered}
$$

Variational inequality (3.23) can be considered at $t=0$ which provides the uniqueness of the displacement for $t=0$. Hence $\operatorname{div} u(0)=0$ and, consequently, equation (3.28) implies

$$
\delta^{2} \operatorname{div} u(t)=-\theta(t)+\int_{0}^{t} \Delta \theta .
$$


Substitution of this value of $\delta^{2} \operatorname{div} u$ in (3.29) provides the inequality

$$
\int_{\Omega_{c}} \sigma_{i j}(u) \varepsilon_{i j}(u)-\int_{\Omega_{c}} \theta\left(-\theta+\int_{0}^{t} \Delta \theta\right) \leqslant 0,
$$

i.e.

$$
\|u(t)\|_{H^{1}\left(\Omega_{c}\right)}^{2}+\|\theta(t)\|_{L^{2}\left(\Omega_{c}\right)}^{2}+\frac{1}{2} \frac{\mathrm{d}}{\mathrm{d} t}\left\|\int_{0}^{t} \nabla \theta\right\|_{L^{2}\left(\Omega_{c}\right)}^{2} \leqslant 0 .
$$

Hence $\theta \equiv 0, u \equiv 0$ which proves the assertion. The solution of (3.12)-(3.18) satisfies the following inclusions:

$$
\theta_{t} \in L^{2}\left(Q_{c}\right), \quad \theta \in L^{2}\left(0, T ; H^{1}\left(\Omega_{c}\right)\right), \quad u \in H^{1}\left(0, T ; H^{1}\left(\Omega_{c}\right)\right) .
$$

Actually, the function $\theta$ has a higher regularity. To see this we write the equation (3.22) in $Q_{c}$ in the following form:

$$
-\Delta \theta=-\theta_{t}-\delta^{2} \frac{\partial}{\partial t} \operatorname{div} u+\gamma(\bar{\theta})|\nabla \varphi|^{2}
$$

with the right-hand side $-\theta_{t}-\delta^{2} \frac{\partial}{\partial t} \operatorname{div} u+\gamma(\bar{\theta})|\nabla \varphi|^{2}$ belonging to $L^{2}(Q)$. Of course, the derivative $\frac{\partial}{\partial t} \operatorname{div} u$ is defined with respect to the domain $Q_{c}$. Conditions (3.17) provide that the equation (3.30) holds in $Q$. In this case we can argue as in the case of the boundary value problem (3.1)-(3.3) which, in fact, removes the singularity surface $\Xi \times(0, T)$. Consequently, by the boundary condition (3.16), we have $\theta \in L^{2}\left(0, T ; H^{2}(\Omega) \cap H_{0}^{1}(\Omega)\right)$. Note that the estimate

$$
\|\theta\|_{L^{2}\left(0, T ; H^{2}(\Omega) \cap H_{0}^{1}(\Omega)\right)} \leqslant c_{6}
$$

for the solution to the system (3.21), (3.22) is also independent of the norm $\|\bar{\theta}\|_{L^{2}\left(0, T ; L^{2}\left(\Omega_{c}\right)\right)}$. The constant $c_{6}$ depends on $Q$ and the $L^{2}$-norm of the right-hand side of (3.30). Notice next that the space

$$
\theta_{t} \in L^{2}\left(0, T ; L^{2}(\Omega)\right), \quad \theta \in L^{2}\left(0, T ; H^{2}(\Omega)\right)
$$

is compactly embedded in the space $\theta \in L^{2}(Q)$ and, consequently, into the space $L^{2}\left(Q_{c}\right)$. This means that if $\bar{\theta}$ belongs to some ball $B_{R}$ in the space $L^{2}\left(0, T ; L^{2}\left(\Omega_{c}\right)\right)$, i.e.

$$
\|\bar{\theta}\|_{L^{2}\left(0, T ; L^{2}\left(\Omega_{c}\right)\right)} \leqslant \mathbb{R}
$$

for $\mathbb{R}$ sufficiently large, the solution $\theta$ belongs to the same ball, and the mapping $A$ : $L^{2}\left(0, T ; L^{2}\left(\Omega_{c}\right)\right) \ni \bar{\theta} \longrightarrow \theta \in L^{2}\left(0, T ; L^{2}\left(\Omega_{c}\right)\right)$ is compact. To use the Schauder fixed point theorem we have to verify a continuity of the mapping $A$ from $L^{2}\left(Q_{c}\right)$ into $L^{2}\left(Q_{c}\right)$. Let $\bar{\theta}^{n} \rightarrow \bar{\theta}$ in $L^{2}\left(Q_{c}\right)$. We shall state that

$$
A\left(\bar{\theta}^{n}\right) \rightarrow A(\bar{\theta}) \text { in } L^{2}\left(Q_{c}\right) .
$$

Denote $\theta^{n}=A\left(\bar{\theta}^{n}\right), \theta=A(\bar{\theta})$. For $\bar{\theta}^{n}$ and $\bar{\theta}$ we can define $\gamma\left(\bar{\theta}^{n}\right)\left|\nabla \varphi^{n}\right|^{2}, \gamma(\bar{\theta})|\nabla \varphi|^{2}$, and solve the problem (3.21), (3.22) which provides an existence of $\left(\theta^{n}, u^{n}\right)$ and $(\theta, u)$, respectively. The functions $\varphi^{n}$ and $\varphi$ satisfy the following equations in $Q$ :

$$
\operatorname{div}\left(\gamma\left(\bar{\theta}^{n}\right) \nabla \varphi^{n}\right)=0, \quad \operatorname{div}(\gamma(\bar{\theta}) \nabla \varphi)=0 .
$$


From the equations

$$
\begin{aligned}
\theta_{t}^{n}-\Delta \theta^{n}+\delta^{2} \frac{\partial}{\partial t} \operatorname{div} u^{n} & =\gamma\left(\bar{\theta}^{n}\right)\left|\nabla \varphi^{n}\right|^{2} \\
\theta_{t}-\Delta \theta+\delta^{2} \frac{\partial}{\partial t} \operatorname{div} u & =\gamma(\bar{\theta})|\nabla \varphi|^{2}
\end{aligned}
$$

it follows that

$$
\delta^{2} \frac{\partial}{\partial t} \operatorname{div}\left(u-u^{n}\right)=-\left(\theta-\theta^{n}\right)_{t}+\Delta\left(\theta-\theta^{n}\right)+\gamma(\bar{\theta})|\nabla \varphi|^{2}-\gamma\left(\bar{\theta}^{n}\right)\left|\nabla \varphi^{n}\right|^{2} .
$$

Since the initial conditions for $\theta$ and $\theta^{n}$ coincide, i.e.

$$
\theta(0)-\theta^{n}(0)=0
$$

equation (3.32) implies

$$
\delta^{2} \operatorname{div}\left(u-u^{n}\right)(t)=-\left(\theta-\theta^{n}\right)(t)+\int_{0}^{t} \Delta\left(\theta-\theta^{n}\right)+\int_{0}^{t}\left[\gamma(\bar{\theta})|\nabla \varphi|^{2}-\gamma\left(\bar{\theta}^{n}\right)\left|\nabla \varphi^{n}\right|^{2}\right] .
$$

Variational inequalities

$$
\begin{array}{cc}
\int_{\Omega_{c}} \sigma_{i j}\left(u^{n}\right) \varepsilon_{i j}\left(\bar{u}-u^{n}\right)-\delta^{2} \int_{\Omega_{c}} \theta^{n} \operatorname{div}\left(\bar{u}-u^{n}\right) \geqslant \int_{\Gamma_{2}} g_{i}\left(\bar{u}_{i}-u_{i}^{n}\right) & \forall \bar{u} \in \tilde{K}, \\
\int_{\Omega_{c}} \sigma_{i j}(u) \varepsilon_{i j}(\bar{u}-u)-\delta^{2} \int_{\Omega_{c}} \theta \operatorname{div}(\bar{u}-u) \geqslant \int_{\Gamma_{2}} g_{i}\left(\bar{u}_{i}-u_{i}\right) & \forall \bar{u} \in \tilde{K}
\end{array}
$$

provide the relation

$$
\int_{\Omega_{c}} \sigma_{i j}\left(u-u^{n}\right) \varepsilon_{i j}\left(u-u^{n}\right)-\delta^{2} \int_{\Omega_{c}}\left(\theta-\theta^{n}\right) \operatorname{div}\left(u-u^{n}\right) \leqslant 0
$$

which, by (3.33), gives

$$
\begin{gathered}
\int_{\Omega_{c}} \sigma_{i j}\left(u-u^{n}\right) \varepsilon_{i j}\left(u-u^{n}\right) \\
+\int_{\Omega_{c}}\left(\theta-\theta^{n}\right)\left(\left(\theta-\theta^{n}\right)-\int_{0}^{t} \Delta\left(\theta-\theta^{n}\right)-\int_{0}^{t}\left[\gamma(\bar{\theta})|\nabla \varphi|^{2}-\gamma\left(\bar{\theta}^{n}\right)\left|\nabla \varphi^{n}\right|^{2}\right]\right) \leqslant 0 .
\end{gathered}
$$

Consequently, (3.34) implies the following inequality holding for almost all $t \in(0, T)$ :

$$
\begin{gathered}
\left\|u-u^{n}\right\|_{H^{1}\left(\Omega_{c}\right)}^{2}+\left\|\theta-\theta^{n}\right\|_{L^{2}\left(\Omega_{c}\right)}^{2}+\frac{1}{2} \frac{\mathrm{d}}{\mathrm{d} t}\left\|\int_{0}^{t} \nabla\left(\theta-\theta^{n}\right)\right\|_{L^{2}\left(\Omega_{c}\right)}^{2} \\
\leqslant \int_{0}^{t} \int_{\Omega_{c}}\left[\gamma(\bar{\theta})|\nabla \varphi|^{2}-\gamma\left(\bar{\theta}^{n}\right)\left|\nabla \varphi^{n}\right|^{2}\right]\left(\theta-\theta^{n}\right) .
\end{gathered}
$$

Integrating (3.35) in $t$ we derive

$$
\int_{0}^{T}\left\|u-u^{n}\right\|_{H^{1}\left(\Omega_{c}\right)}^{2}+\left\|\theta-\theta^{n}\right\|_{L^{2}\left(Q_{c}\right)}^{2} \leqslant T \int_{Q_{c}}\left[\gamma(\bar{\theta})|\nabla \varphi|^{2}-\gamma\left(\bar{\theta}^{n}\right)\left|\nabla \varphi^{n}\right|^{2}\right]\left(\theta-\theta^{n}\right) .
$$


Since

$$
\begin{gathered}
T \int_{Q_{c}}\left[\gamma(\bar{\theta})|\nabla \varphi|^{2}-\gamma\left(\bar{\theta}^{n}\right)\left|\nabla \varphi^{n}\right|^{2}\right]\left(\theta-\theta^{n}\right) \\
\leqslant(1 / 2)\left\|\theta-\theta^{n}\right\|_{L^{2}\left(Q_{c}\right)}^{2}+c \int_{Q_{c}}\left[\gamma(\bar{\theta})|\nabla \varphi|^{2}-\gamma\left(\bar{\theta}^{n}\right)\left|\nabla \varphi^{n}\right|^{2}\right]^{2}
\end{gathered}
$$

from (3.36) it follows that

$$
\left\|\theta-\theta^{n}\right\|_{L^{2}\left(Q_{c}\right)}^{2} \leqslant c \int_{Q_{c}}\left[\gamma(\bar{\theta})|\nabla \varphi|^{2}-\gamma\left(\bar{\theta}^{n}\right)\left|\nabla \varphi^{n}\right|^{2}\right]^{2} .
$$

Now we want to prove that the right-hand side of (3.37) goes to zero as $n \rightarrow \infty$. Note that

$$
\gamma(\bar{\theta})|\nabla \varphi|^{2}-\gamma\left(\bar{\theta}^{n}\right)\left|\nabla \varphi^{n}\right|^{2}=\left(\gamma(\bar{\theta})-\gamma\left(\bar{\theta}^{n}\right)\right)|\nabla \varphi|^{2}+\gamma\left(\bar{\theta}^{n}\right)\left(\nabla\left(\varphi-\varphi^{n}\right) \cdot \nabla\left(\varphi+\varphi^{n}\right)\right) .
$$

Hence

$$
\begin{gathered}
\int_{Q_{c}}\left[\gamma(\bar{\theta})|\nabla \varphi|^{2}-\gamma\left(\bar{\theta}^{n}\right)\left|\nabla \varphi^{n}\right|^{2}\right]^{2} \\
\leqslant 2 \int_{Q_{c}}\left(\gamma(\bar{\theta})-\gamma\left(\bar{\theta}^{n}\right)\right)^{2}|\nabla \varphi|^{4}+2 \gamma_{2}^{2} \int_{Q_{c}}\left|\nabla\left(\varphi-\varphi^{n}\right)\right|^{2} \cdot\left|\nabla\left(\varphi+\varphi^{n}\right)\right|^{2} .
\end{gathered}
$$

Since $\theta^{n}$ are relatively compact in some ball $B_{\mathbb{R}} \subset L^{2}\left(Q_{c}\right)$ it suffices to prove that $\theta$ is a unique limit point of $\theta^{n}$. Let $\tilde{\theta}$ be a limit point, i.e. $\tilde{\theta}=\lim _{k \rightarrow \infty} \theta^{n_{k}}$. Choosing a subsequence $\bar{\theta}^{n_{k_{p}}}$ with the previous notation $\bar{\theta}^{n_{k}}$, we assume that

$$
\bar{\theta}^{n_{k}} \rightarrow \bar{\theta} \quad \text { almost everywhere in } \quad Q_{c} .
$$

We already have proved that

$$
\int_{Q_{c}}|\nabla \varphi|^{4}<c
$$

whence, by the Lebesgue convergence theorem and (3.39), the first integral of the right-hand side of (3.38) goes to zero as $n=n_{k} \rightarrow \infty$. We take below $n=n_{k}$. Now we prove that the second integral of the right-hand side of (3.38) converges to zero as $n \rightarrow \infty$. From (3.31) we have the following equation in $Q$ :

$$
\operatorname{div}\left(\nabla\left(\varphi-\varphi^{n}\right)\right)=\frac{2}{\gamma_{1}+\gamma_{2}} \operatorname{div}\left[\left(\gamma\left(\bar{\theta}^{n}\right)-\gamma(\bar{\theta})\right) \nabla \varphi\right]-\frac{2}{\gamma_{1}+\gamma_{2}} \operatorname{div}\left[\left(\tilde{\gamma}\left(\bar{\theta}^{n}\right) \nabla\left(\varphi-\varphi^{n}\right)\right] .\right.
$$

Since $\left|\tilde{\gamma}\left(\bar{\theta}^{n}\right)\right| \leqslant \frac{\gamma_{2}-\gamma_{1}}{2}$, by (2.10) and the boundary condition

$$
\varphi-\varphi^{n}=0 \quad \text { on } \quad \Gamma \times(0, T),
$$

from (3.40) we obtain the following estimate:

$$
\left\|\nabla\left(\varphi-\varphi^{n}\right)\right\|_{L^{4}(\Omega)} \leqslant \frac{2 \Lambda_{4}}{\gamma_{1}+\gamma_{2}}\left\|\left(\gamma\left(\bar{\theta}^{n}\right)-\gamma(\bar{\theta})\right) \nabla \varphi\right\|_{L^{4}(\Omega)}+\Lambda\left\|\nabla\left(\varphi-\varphi^{n}\right)\right\|_{L^{4}(\Omega)}
$$


being uniform in $t \in(0, T)$. Recall that $\Lambda<1$, hence there exists a constant $c$ independent of $n$ such that

$$
\left\|\nabla\left(\varphi-\varphi^{n}\right)\right\|_{L^{4}(\Omega)}^{4} \leqslant c\left\|\left(\gamma\left(\bar{\theta}^{n}\right)-\gamma(\bar{\theta})\right) \nabla \varphi\right\|_{L^{4}(\Omega)}^{4} .
$$

Integrating (3.41) in $t$ implies

$$
\left\|\nabla\left(\varphi-\varphi^{n}\right)\right\|_{L^{4}(Q)} \leqslant c\left\|\left(\gamma\left(\bar{\theta}^{n}\right)-\gamma(\bar{\theta})\right) \nabla \varphi\right\|_{L^{4}(Q)} .
$$

In view of (3.39) the right-hand side of (3.42) goes to zero by the Lebesgue convergence theorem, hence

$$
\left\|\nabla\left(\varphi-\varphi^{n}\right)\right\|_{L^{4}(Q)} \rightarrow 0, \quad n \rightarrow \infty .
$$

Since $\left\|\nabla\left(\varphi+\varphi^{n}\right)\right\|_{L^{4}(Q)}$ are bounded uniformly in $n$, the convergence (3.43) yields as $n \rightarrow \infty$

$$
\int_{Q_{c}}\left|\nabla\left(\varphi-\varphi^{n}\right)\right|^{2} \cdot\left|\nabla\left(\varphi+\varphi^{n}\right)\right|^{2} \leqslant\left\|\nabla\left(\varphi-\varphi^{n}\right)\right\|_{L^{4}\left(Q_{c}\right)}^{2}\left\|\nabla\left(\varphi+\varphi^{n}\right)\right\|_{L^{4}\left(Q_{c}\right)}^{2} \rightarrow 0 .
$$

Therefore, we conclude that the second integral of the right-hand side of (3.38) converges to zero, and we prove that

$$
\theta^{n_{k}} \rightarrow \theta=\tilde{\theta} \quad \text { in } \quad L^{2}\left(Q_{c}\right) .
$$

Continuity of the operator $A$ is established. So we can apply the Schauder fixed point theorem to assure the existence of a solution to the problem (2.1)-(2.9) in the sense of Theorem 2.1.

\section{Concluding remarks}

We have some additional regularity for the solution of (2.12)-(2.16): in particular, $\theta \in$ $L^{2}\left(0, T ; H^{2}(\Omega) \cap H_{0}^{1}(\Omega)\right)$. This inclusion follows from the equation

$$
-\Delta \theta=-\theta_{t}-\delta^{2} \frac{\partial}{\partial t} \operatorname{div} u+\gamma(\theta)|\nabla \varphi|^{2}
$$

and the given boundary conditions for $\theta$ on $\Gamma \times(0, T)$ and $\Xi \times(0, T)$. Recall that the boundary conditions on $\Xi \times(0, T)$ remove the singularity surface $\Xi \times(0, T)$.

Boundary conditions (2.6), (2.9) are included in the variational inequality (2.14). It can be shown (see [10]) that the displacement $u$ also has an additional regularity, at least in the case when $\Xi$ is a straight line segment. In particular, for any $x \in \Xi$ there exists a neighbourhood $V$ such that $u \in L^{2}\left(0, T ; H^{2}\left(V \cap \Omega_{c}\right)\right)$. Consequently, from the variational inequality (2.14) it follows that boundary conditions (2.9) hold for almost all $(x, t) \in \Xi \times(0, T)$.

We can state an additional smoothness properties of $\varphi$ provided that $\gamma$ is $C^{1}$-function such that $\left|\gamma^{\prime}(s)\right|<\gamma_{3}, \gamma_{3}=$ const. Namely,

$$
\varphi \in L^{q}\left(0, T ; H^{2}(\Omega)\right), \quad q<4 .
$$

Indeed, equation (2.16) reads

$$
\Delta \varphi=-\frac{\gamma^{\prime}(\theta)}{\gamma(\theta)} \nabla \theta \cdot \nabla \varphi \quad \text { in } \quad Q .
$$


According to [16] the space

$$
\theta_{t} \in L^{2}\left(0, T ; L^{2}(\Omega)\right), \quad \theta \in L^{2}\left(0, T ; H^{2}(\Omega)\right)
$$

has (compact) embedding in the space $\theta \in L^{q}\left(0, T ; H^{\frac{3}{2}}(\Omega)\right), q<4$.

Consider the right-hand side of equation (4.2). Since the embedding $H^{1 / 2}(\Omega) \subset L^{4}(\Omega)$ is continuous for the two-dimensional case we have

$$
\nabla \theta \in L^{q}\left(0, T ; L^{4}(\Omega)\right) .
$$

Consequently, by the inclusion $\nabla \varphi \in L^{\infty}\left(0, T ; L^{4}(\Omega)\right)$ and (4.3), we have $\nabla \varphi \cdot \nabla \theta \in$ $L^{q}\left(0, T ; L^{2}(\Omega)\right)$. The right-hand side of equation (4.2) belongs to $L^{q}\left(0, T ; L^{2}(\Omega)\right)$, and $\Phi_{0} \in$ $L^{\infty}\left(0, T ; H^{2}(\Omega)\right)$, hence the inclusion (4.1) follows.

Also, note that to prove the theorem it suffices to require a weaker regularity assumption on $\varphi_{0}$. We can assume that $\varphi_{0}$ is a trace on $\Gamma \times(0, T)$ of a function $\Phi_{0} \in L^{\infty}\left(0, T ; W_{4}^{1}(\Omega)\right)$.

\section{Acknowledgements}

A. M. Khludnev was supported by the Russian Fund for Basic Research (grant 00-01-00842). The project this paper is based on is supported by the Federal Ministry of Education and Research of Germany under grant 03HO7FV1/0.

\section{REFERENCES}

1. Ames, K. A. \& PAYNE, L. E. Uniqueness and continuous dependence of solutions to a multidimensional thermoelastic contact problem. J. Elast. 34, (1994) 139-148.

2. AndREWS, K. T. \& ShILlor, M. A parabolic initial-boundary value problem modelling axially symmetric thermoelastic contact. Nonlinear Anal. 22, (1994) 1529-1551.

3. Andrews, K. T., Shillor, M., \& Wright, S. A hyperbolic-parabolic system modelling the thermoelastic impact of two rods. Math. Methods Appl. Sci. 17, (1994) 901-918.

4. Antontsev, S. N. \& Chipot, M. The thermistor problem: existence, smoothness, uniqueness, blowup. SIAM J. Math. Anal. 25, (1994) 1128-1156.

5. Снipot, M. \& Cimatti, G. A uniqueness result for the thermistor problem. Eur. J. Appl. Math. 2, (1991) 97-103.

6. Dautray, R. \& Lions, J.-L. Mathematical Analysis and Numerical Methods for Science and Technology. vol. 1, Springer, Berlin (1990).

7. Dreyer, W., Duderstadt, F., \& Hömberg, D. Finite element simulation des widerstandsimpulsschweissens. Technische Mechanik 19, (1999) 331-340.

8. Howison, S., Rodrigues, J. F., \& Shillor, M. Stationary solutions to the thermistor problem. $J$. Math. Anal. Appl. 174, (1993) 573-588.

9. Khludnev, A. M. The contact problem for a shallow shell having the crack. Appl. Math. Mech. 59, (1995) 299-306.

10. Khludnev, A. M. The equilibrium problem for a thermoelastic plate with a crack. Siberian Math. J. 37, (1996) 394-404.

11. Khludnev, A. M. Contact problem for a plate having a crack of minimal opening. Control Cybern. 25, (1996) 605-620.

12. Khludnev, A. M. \& Sokolowski, J. Modelling and Control in Solid Mechanics. Birkhauser, Basel (1997).

13. NOWACKI, W. Problems of Thermoelasticity. USSR Acad. Sci., Moscow (1962), in Russian. 
14. SHI, P. \& ShILlor, M. Existence of a solution to the $N$-dimensional problem of thermoelastic contact. Commun. Partial Diff. Eqns 17, (1992) 1597-1618.

15. Shi, P., Shillor, M., \& XU, X. Existence of a solution to the Stefan problem with Joule's heating. J. Diff. Eqns 105, (1993) 239-263.

16. Simon, J. Compact sets in the space $L^{p}(0, T ; B)$. Ann. Mat. Pura Appl. (4) 146, (1987) 65-96.

17. Troianiello, G. M. Elliptic Differential Equations and Obstacle Problems. Plenum, New York (1987).

18. $\mathrm{XU}, \mathrm{X}$. The $N$-dimensional quasistatic problem of thermoelastic contact with Barber's heat exchange conditions. Adv. Math. Sc. Appl. 6, (1996) 559-587.

19. YUAN, G. W. Regularity of solution of the thermistor problem. Appl. Anal. 53, (1994) 149-155.

20. YUAN, G. W. \& LIU, Z. H. Existence and uniqueness of the $C^{\alpha}$ solution for the thermistor problem with mixed boundary value. SIAM J. Math. Anal. 25, (1994) 1157-1166. 